\title{
Immune factors and thyroid function parameters associated with different degrees anemia in patients living with HIV/AIDS in Southwest China: a cross-sectional study
}

\section{Liling Huang}

Chest Hospital of Guangxi Zhuang Autonomous Region

\section{Bo Xie}

Guangxi Medical University

\section{Kai Zhang}

Chest Hospital of Guangxi Zhuang Autonomous Region

\section{Yuanlong $\mathrm{Xu}$}

Chest Hospital of Guangxi Zhuang Autonomous Region

\section{Lingsong Su}

Chest Hospital of Guangxi Zhuang Autonomous Region

Yu Lv

Chest Hospital of Guangxi Zhuang Autonomous Region

Yangjie Lu

Chest Hospital of Guangxi Zhuang Autonomous Region

\section{Xianwu Pang}

Guangxi Medical University

\section{Hong Qiu}

Guangxi Medical University

\section{Lanxiang Li}

Guangxi Medical University

\section{Xihua Wei}

Guangxi Medical University

Yanling Hu ( $\nabla$ ylhupost@163.com )

Guangxi Medical University https://orcid.org/0000-0001-5753-1601

Jiannan LV

Chest Hospital of Guangxi Zhuang Autonomous Region

\section{Research Article}


Keywords: HIV, AIDS, CD4+ T cell counts, Immune, Anemia

Posted Date: February 23rd, 2022

DOI: https://doi.org/10.21203/rs.3.rs-1349757/v1

License: (c) (1) This work is licensed under a Creative Commons Attribution 4.0 International License. Read Full License 


\section{Abstract}

Background: In the era of HIV/AIDS antiretroviral therapy, the prevalence of anemia in HIV/AIDS is very high, but the factors associated with anemia are not yet clear. In order to actively prevent HIV/AIDS patients from having anemia, we investigated the immune indexes of HIV/AIDS patients complicated with anemia, and discussed the immunological risk factors associated with anemia in HIV/AIDS patients.

Methods: We collected demographic information and clinical data about patient living with HIV/AIDS from January 2016 to October 2021 from the hospital information system. Logistic regression models was used to analyze these data to identify factors associated with different anemia.

Results: At last, 1131 AIDS patients were enrolled in the study, including men (72.5\%) and women $(27.5 \%)$, and the age was mainly concentrated in $>40$ years $(85.94 \%)$. The overall prevalence of anemia in Guangxi was $26.9 \%$ (95\% Cl: $24.2 \%-29.6 \%$ ). The factors for affecting anemia were CD4 ${ }^{+} \mathrm{T}$ cell count $\leq$ 200 cell/ul (aOR: 5.523, 95\% Cl: 2.217, 13.749), CD4 / CD8 (aOR: 3.827, 95\% Cl: 1.669, 8.785 ), free thyroxine [FT4] (aOR: $10.612,95 \% \mathrm{Cl}: 2.03,55.479)$, globulin (aOR: $1.027,95 \% \mathrm{Cl}: 1.003,1.052)$ and thyroid hormone [T4] (aOR: 0.795, 95\% Cl: 0.67, 0.943).

Conclusion: Immune factors and thyroid related hormone were associated with different anemia in patients living with HIV/AIDS. Monitoring these factors timely will be helpful for treatment and improving the quality of life and life expectancy of patients.

\section{Background}

AIDS is a highly harmful infectious disease, caused by infection with HIV that can attack the human immune system. This virus takes the most important $C D 4+T$ lymphocytes in the human immune system as the main target, destroying the cells and losing the human immune function. The infected persons are easy to accompany a variety of diseases, and the case fatality rate is higher ${ }^{[1]}$. Over the past 30 years, HIV has put a huge burden on global wealth and health. The WHO estimates that of the year 2020, 37.7 million people were infected with HIV worldwide ${ }^{[2]}$. More than 6 million HIV infections worldwide are on treatment with over 150 antiretroviral drugs ${ }^{[3]}$. The survival of HIV individuals treated with ART improved that ART can reduce viral load and delaye disease progression ${ }^{[4]}$. However, HIV-associated HAND, CVD, MS, and non-HIV-associated malignancies remain the main complications of HIV infection,

which can seriously reduce the quality of life of patients with $\operatorname{AIDS}^{[5]}$. Hematological change is a common complication in individuals infected with HIV/AIDS.

Anaemia can cause a range of symptoms including fatigue, weakness, dizziness and drowsiness ${ }^{[6]}$.Anemia and thrombocytopenia are the most common multifactorial hematologic abnormalities in HIV/AIDS, associated with low quality of life and high mortality ${ }^{[7]}$. Anemia occurs in $20 \%-80 \%$ of people infected with HIV and is one of the important causes of health loss. The prevalence of anemia is clearly associated with the disease progression of HIV/AIDS ${ }^{[8]}$. During the treatment of 
HIV/AIDS patients, drug influences, viral infection and malignant cell infection will affect the blood health system of the patient, and it is very likely to induce anemia ${ }^{[9]}$. However, the related factors affecting the blood system and causing anemia are complex and diverse, which lead to a very difficult treatment of anemia for AIDS patients ${ }^{[10,11]}$.

The diagnostic criteria for anemia of the WHO are less than $110 \mathrm{~g} / \mathrm{L}$ in women and less than $120 \mathrm{~g} / \mathrm{L}$ in men. Anemia was divided into four grades: mild anemia, moderate anemia, severe anemia and extremely severe anemia according to different hemoglobin levels ${ }^{[12]}$. Many risk factors are associated with anemia in AIDS patients. For example, older age, lower CD4 counts, and ethnic minorities were associated with an increased risk of anemia ${ }^{[13]}$; Anemia in AIDS patients was also highly associated with no ART, a history of anti-tuberculosis medication, an ART regimen containing zidovudine (ZDV), and a CD $4+\mathrm{T}$ lymphocyte count $\leq 200$ cell/[ ${ }^{[14]}$. Additionally, ART also seems to accelerate anemia in some cases ${ }^{[15]}$. The pathogenesis of anemia in AIDS/HIV patients was related with many etiological factors. However, limited information were found for the correlated causes immune factors.

Studies have proved that anemia is related to the immune state of the body ${ }^{[34]}$. HIV mainly destroys the immune system of infected people and causes the disorder of the immune system. The immune system mediates many diseases of the blood system ${ }^{[35]}$. The three aspects interact with each other and the relationship is complex. It leads to accelerated disease progression and decreased quality of life. Paying attention to the changes of immune factors in HIV infected patients with anemia can promote the good prognosis of the disease.

\section{Materials And Methods}

\subsection{Study Design and Settings}

A cross-sectional study was conducted in Guangxi Chest Hospital, Liuzhou City, Guangxi Zhuang Autonomous Region, China. Guangxi Chest Hospital is the unique provincial hospital and the HIV/AIDS prevention and control clinical center in Guangxi. We extracted the demographic, laboratory examination results and diagnostic information of all hospitalized HIV infected patients from 2016 to 2021 from the hospital's information system. The anemia prevalence rate of HIV infected people in the hospital is counted according to the year, and the results are shown in Fig. 1. Among all hospitalized HIV infected patients in our hospital, the overall prevalence of anemia was $26.9 \%$ (95Cl: $24.2 \%-29.6 \%$ ). Among them, more than half $(52.7 \%, n=966)$ had moderate anemia, while more than one quarter $(30.6 \%, n=560)$ had severe anemia. The prevalence of anemia in AIDS patients is high.

\subsection{Grouping and inclusion criteria}

A total of 6882 patients were confirmed to be HIV antibody positive by laboratory examination. Among them, cases of missing individual information $(n=231)$, cases of missing laboratory examination results $(n=5244)$ and patients with hereditary and secondary anemia $(n=27)$ were excluded from the study. 
Finally, a total of 1131 patients with HIV infection complicated with anemia were included in this study.According to the WHO definition of anemia ${ }^{[11]}$, the cases included in the study were divided into four categories: non anemia $(n=753)$, mild anemia $(n=90)$, moderate anemia $(n=184)$ and severe anemia $(n=104)$ (Fig. 2).

\subsection{Study variables}

The sociodemographic characteristics determined from the hospital information system include age, gender, residence, nationality and marital status. In addition, the clinical and biochemical test characteristics of patients collected from laboratory examination results include $\mathrm{CD}^{+} \mathrm{T}$ cell count (cell/ul), CD $4^{+} \mathrm{T}$ cell count (cell/ul), $\mathrm{CD} 45^{+} \mathrm{T}$ cell count (cell/ul), CD8 ${ }^{+} \mathrm{T}$ cell count (cell/ul), CD4/CD8, neutrophil count $\left(10^{\wedge} 9 / \mathrm{L}\right)$, thymidine [T4] $(\mathrm{ug} / \mathrm{dl})$, triiodothyronine [T3] $(\mathrm{ng} / \mathrm{ml})$, free thyroxine [FT4] $(\mathrm{ng} / \mathrm{dL})$, free triiodothyronine $[\mathrm{FT} 3](\mathrm{pg} / \mathrm{mL})$, lymphocyte count $\left(10^{\wedge} 9 / \mathrm{L}\right)$, total lymphocytes, globulin $(\mathrm{g} / \mathrm{L})$, WBC count (10^9/L). The CD4 T-lymphocyte was determined based on WHO recommendation of 2007[23] and the China AIDS treatment guideline (2018 Edition) ${ }^{[24]}$ to commence ART for HIV positive peoples .

\subsection{Data Analysis}

Using Python version 3.9 to extract, organize and clean data. After thorough cleaning, the data were exported to SPSS version 24 statistical software package for further analysis. Descriptive statistics such as median, interquartile range (IQR), frequency and percentage were used to describe the variables of this study. Kruskal Wallis test and chi-square test were used to compare the differences between variables in patients with different anemia grades. To describe the anemia related factors in HIV/AIDS, logistic regression model was used to calculate $95 \% \mathrm{Cl}$ odds ratio.This statistical test was a two-sided test with a level of statistical significance of 0.05 .

\section{Result}

\subsection{Characteristics of study participants}

A total of 1131 cases were included. The median age was 56 (IQR:46-66). The majority (72.5\%) of the study participants were men. More than half $(53.58 \%)$ of the study participants were ethnic minorities. More than three quarters $(83.11 \%)$ of the subjects came from rural residential areas. More than half (58.97\%) of the study participants were married. Table 1 summarizes the detailed characteristics of study participants.

\subsection{The clinical and biochemical tests characteristics}

In order to explore the related immune factors of AIDS patients with anemia, we analyzed the related immune factors, and the results of single factor analysis of related immune factors are shown in Table 2. The results showed that: $C D 3^{+} T$ cells $\left(X^{2}=23.482, P<0.01\right), C D 4^{+} T$ cells $\left(X^{2}=18.432, P=\right.$ $0.005), C D 45^{+} T$ cells $\left(X^{2}=27.419, P<0.01\right), C D 8^{+} T$ cells $\left(X^{2}=16.128, P=0.001\right)$, neutrophils $\left(X^{2}=\right.$ 
11.391, $P=0.01)$, thyroxine [T4] $\left(X^{2}=19.16, P<0.01\right)$, triiodothyronine [T3] $\left(X^{2}=34.266, P<0.01\right)$, free thyroxine $[F T 4]\left(X^{2}=30.502, P<0.01\right)$ Free three iodine methyl $[F T 3]\left(X^{2}=30.416, P<0.01\right)$, globulin $\left(X^{2}=\right.$ $27.419, P<0.01)$ and WBC $\left(X^{2}=27.419, P<0.01\right)$ have significant statistical differences, suggesting that the progression of anemia in AIDS patients may be significantly associated to the above factors.

\subsection{Adjusted ordered multiple regression analysis results}

An analysis of the immune variables associated with anemia in HIV/AIDS was conducted by sequential logistic regression analysis. The results are shown in Table 3. A total of 14 immune related variables were included in the logistic regression model. Finally, $C D 4^{+} \mathrm{T}$ cell count, CD4/CD8, thyroid hormone[T4], free thyroxine[FT4], globulin[GLOB] entered the adjusted regression model. Among them, $\mathrm{CD} 4^{+} \mathrm{T}$ cell count $\leq$ $200 \mathrm{cell} / \mathrm{ul}([\mathrm{aOR}]=5.523[95 \% \mathrm{Cl}: 2.217,13.749], \mathrm{P}<0.01), \mathrm{CD} 4 / \mathrm{CD} 8(\mathrm{aOR}=3.827[95 \% \mathrm{Cl}=1.669,8.785]$, $\mathrm{P}=0.002)$, free thyroxine [FT4] $(\mathrm{aOR}=10.612[95 \% \mathrm{Cl}: 2.03,55.479], \mathrm{P}=0.005)$, and globulin $(\mathrm{aOR}=$ $1.027[95 \% \mathrm{Cl}: 1.003,1.052], \mathrm{P}=0.026)$ were all risk factors for anemia. As a sensitive variable to detect thyroid function, thyroid hormone $[\mathrm{T} 4]$ ( $\mathrm{aOR}=0.795[95 \% \mathrm{Cl}: 0.67,0.943], \mathrm{P}=0.008)$ is a protective factor. It can be seen that anemia has a negative impact on the quality of life and the disease progress of HIV/AIDS.

\section{Discussion}

Anemia is an important clinical symptom of HIV/AIDS and is associated with HIV disease progression, decreased survival, and low life quality Additionally, anemia is also a common medical problem affecting people living with HIV/AIDS by complicating the pathogenesis of the disease ${ }^{[25]}$. A number of literatures indicate that immune factors are closely related with the anemia. Infection with HIV-1 leads to a dramatic depletion of $\mathrm{CD}^{+} \mathrm{T}$ cells and an altered distribution of T-cell subsets. In view of complicated immune regulation in HIV patient, the profiling of immune factors may be different effection on anemia. In our study, a hospital based cross-sectional study was used to investigate the immune factors related to different degrees anemia in HIV/AIDS. The main results showed that CD $4^{+} \mathrm{T}$ cell $\leq 200 \mathrm{cell} / \mathrm{ul}$, higher $\mathrm{CD} 4 / \mathrm{CD}$ 8, decreased thyroid hormone [T4], elevated free thyroxine [FT4] and elevated globulin were associated with increased risk of anaemia. At the same time, we found that the prevalence of anemia was $26.9 \%$. This figure is lower compared to previous study (the prevalence is $51.9 \%{ }^{[12]}$. Many studies have underlined that CD4/CD8 ratio might represent a good predictor of AIDS and non-AIDS events ${ }^{[26]}$. Low or high CD4/CD8 ratio means immune dysfunction. HIV/AIDS usually leads to CD4/CD8 inversion. [27]. However, our study found that patients with elevated CD4/CD8 were more likely to be complicated with anemia. This means that patients with anemia are in a situation of hyperactivity of autoimmune state, and autoimmune hemolytic anemia (AIHA) is one of the rare complications of HIV/AIDS. Because AIDS patients with AlHA may have a fatal reaction to red blood cell transfusion ${ }^{[17]}$, Several studies have demonstrated a clear association between $\mathrm{CD} 4^{+} \mathrm{T}$ cell counts and hematological abnormalities in patients with HIV/AIDS ${ }^{[14]}$. Lower $\mathrm{CD} 4^{+} T$ cell counts were also previously considered as a risk factor for anemia ${ }^{[15,16]}$. This study shows that patients with $C D 4^{+} T$ cell counts $\leq 200$ cell/ul are more likely to develop anemia that is inconsistent with the results of CD4/CD8. At the same time, some studies indicate 
that $\mathrm{CD} 4 / \mathrm{CD} 8$ ratio is increasingly becoming a valuable marker of immune activation and immune aging $^{[32,33]}$.

In this study, we found that HIV infected individuals with lower thyroid hormone [T4] had a higher risk of anemia. Thyroid hormone [T4] is a sensitive indicator for detecting thyroid function, and its reducing secretion would impaired thyroid function in patients with AIDS. Anemia caused by reduced thyroid function is reported in several diseases ${ }^{[18]}$. Mainly because thyroxine [T4] can partially affect iron absorption and reduce the metabolic rate of hematopoietic tissue, and decrease tissue oxygen consumption in plasma and within red blood cells that leads to the reduction of erythropoietin secretion [29]. Previous studies have found that certain autoimmune processes can lead to malignant anemia and thyroid disease ${ }^{[19]}$. This further proves that HIV infected people in a hyperimmune state are more prone to anemia. Our study showed that the increased secretion of free thyroxine [FT4] leaded to an increased risk of anemia. Free thyroxine [FT4] is a hormone with real physiological activity. The concentration of free thyroxine [FT4] in the human body not only reflects the thyroid function, but also the lesions of other organs (mainly related to liver and kidney) are closely related with the altered concentration of free thyroxine $[\mathrm{FT} 4]^{[30]}$. Through carrying out the association between free thyroxine [FT4] and anemia, some studies have demonstrated a clear correlation between serum free thyroxine [FT4] and serum hemoglobin $[\mathrm{Hb}]^{[20]}$. However, the detailed mechanism of anemia in HIV infected patients caused by free thyroxine [FT4] remains to be further explored.

Additionally, elevated globulin was also found to associate with increased risk of anemia in HIV patients. Globulin is mainly secreted by plasma cells, and its main function is involved in enhancing immunity. Some external inflammatory factors and infectious factors will induce the rise of globulin.It has been shown that globulin can serve as a marker for the progression of multiple diseases, including NPC ${ }^{[21]}$, tuberculosis $^{[22]}$ and the COVID-19 infection ${ }^{[23]}$. Different kinds of infection is also one of the influencing factors of HIV/AIDS anemia ${ }^{[31]}$. However, there are few studies on the correlation between anemia and globulin. The detailed affection of anemia to HIV infected patients caused by globulin remains to be further investigated.

\section{Conclusions}

Taken together, we found that patients with elevated CD4/CD8 were more likely to be complicated with anemia. Additionally, other factors such as lower CD4 ${ }^{+} \mathrm{T}$ cell counts, decreased thyroid hormone [T4], elevated free thyroxine [FT4], and elevated globulin were associated with an increased risk of anemia. This study reveals that strengthening the monitoring of immune indicators of AIDS patients can we timely detect the signs of anemia complications and timely take intervention measures, which is of great help to improve the quality of life and life expectancy of patients.

\section{Abbreviations}


AIDS

acquired immunodeficiency syndrome

ART

antiretroviral therapy

HIV

human immunodeficiency virus

WBC

white blood cell

HAND

neurocognitive impairment

CVD

cardiovascular disease

MS

metabolic syndrome

aOR

adjusted odds ratio

WHO

the World Health Organization.

\section{Declarations}

\section{Ethics approval and consent to participate}

This study was approved by the Human Research Ethics Committee of Guangxi Medical University (Ethics Approval Number: 20210172). After we received the relevant data, the purpose of the study has been clearly informed to the professionals working in the hospital. All patient information was desensitized, and patient privacy was not disclosed during the study.

\section{Consent for publication}

Not applicable.

\section{Availability of data and material}

The datasets generated and/or analysed during the current study are not publicly available because of ethical and legal reasons but are available from the corresponding author Yanling Hu on reasonable request.

\section{Competing interests}

The authors declare that they have no financial conflicts of interest.

\section{Funding}


This research was supported by Guangxi Key Research and Development Program (No. Guike AB20059002).

\section{Authors' contributions}

Y. L.H, J.N.L, L.L.H and K.Z: designed the study and provided the correlative knowledge. Y.L.X, K.Z, L.L.H, Y.J.L and J.N.L: collected and provided the data. L.X.L, X.H.W and B.X extracted data and cleaned data. K.Z, L.S.S, Y.L and B.X generated the figures and tables. Y.L.X, Y.L.H ,X.W.P and B.X: wrote and edited the manuscript. All of the authors read and approved the final manuscript.

\section{Acknowledgements}

We would like to express our gratitude to all participants involved in this study and Guangxi Chest Hospital for their support.

\section{References}

1. Yoshimura K.Current status of HIV/AIDS in the ART era.J Infect Chemother, 2017,23(1):12-16.

2. World Health Organization.Number of people (all ages) living with HIV-Estimates by WHO region[EB/OL]. https://apps.who.int/gho/data/view.main.22100WHO?lang=en.Accessed 12 Dec 2020.

3. Miller V, Nwokike J, Stergachis A.Pharmacovigilance and global HIV/AIDS.Curr Opin HIV AIDS, 2012,7(4):299-304.

4. Lu D Y, Wu H Y, Yarla N S, et al.HAART in HIV/AIDS Treatments: Future Trends.Infect Disord Drug Targets, 2018,18(1):15-22.

5. Valka J, Cermak J. Differential diagnosis of anemia. Vnitr Lek,64(5):468-475.

6. Marchionatti A, Parisi M M.Anemia and thrombocytopenia in people living with HIV/AIDS: a narrative literature review.Int Health, 2021,13(2):98-109.

7. Durandt C, Potgieter J C, Mellet J, et al.HIV and haematopoiesis.S Afr Med J, 2019,109(8b):40-45.

8. Adias T C, Uko E, Erhabor O.Anaemia in human immunodeficiency virus infection: a review.Niger $\mathrm{J}$ Med, 2006,15(3):203-206.

9. Van den Berg K, Murphy E L, Pretorius L, et al.The impact of HIV-associated anaemia on the incidence of red blood cell transfusion: implications for blood services in HIV-endemic countries.Transfus Apher Sci, 2014,51(3):10-18.

10. Marti-Carvajal A J, Sola I, Pena-Marti G E, et al.Treatment for anemia in people with AIDS.Cochrane Database Syst Rev, 2011(10):D4776.

11. Nutritional anaemias: tools for effective prevention and control. Geneva: World Health Organization; 2017. Licence: CC BY-NC-SA 3.0 IGO.

12. Shen $Y$, Wang $Z$, Lu H, et al.Prevalence of anemia among adults with newly diagnosed HIV/AIDS in China.PLoS One, 2013,8(9):e73807. 
13. Melese $\mathrm{H}$, Wassie $M \mathrm{M}$, Woldie $\mathrm{H}$, et al.Anemia among adult HIV patients in Ethiopia: a hospitalbased cross-sectional study.HIV AIDS (Auckl), 2017,9:25-30.

14. Bhardwaj S, Almaeen A, Ahmed W F, et al.Hematologic derangements in HIV/AIDS patients and their relationship with the CD 4 counts: a cross-sectional study.Int J Clin Exp Pathol, 2020,13(4):756-763.

15. Agus S I, Merati T P, Bakta I M, et al.High levels of serum IL-6 and serum hepcidin and low CD4 cell count were risk factors of anemia of chronic disease in HIV patients on the combination of antiretroviral therapy.HIV AIDS (Auckl), 2019,11:133-139.

16. Gedefaw $L$, Yemane T, Sahlemariam Z, et al.Anemia and risk factors in HAART naive and HAART experienced HIV positive persons in south west Ethiopia: a comparative study.PLoS One, 2013,8(8):e72202.

17. Olayemi E, Awodu O A, Bazuaye G N. Autoimmune hemolytic anemia in HIV-infected patients: a hospital based study. Ann Afr Med, 2008,7(2):72-76.

18. Soliman A T, De Sanctis V, Yassin M, et al.Chronic anemia and thyroid function.Acta Biomed, 2017,88(1):119-127.

19. Osborne D, Sobczynska-Malefora A.Autoimmune mechanisms in pernicious anaemia \& thyroid disease.Autoimmun Rev, 2015,14(9):763-768.

20. Kim M, Kim B H, Lee H, et al.Association between Serum Free Thyroxine and Anemia in Euthyroid Adults: A Nationwide Study.Endocrinol Metab (Seoul), 2020,35(1):106-114.

21. Zhong $L T$, Wang $H$, Liang $H$ Q, et al.[An elevated pretreatment serum globulin level predicts a poor prognosis of nasopharyngeal carcinoma].Nan Fang Yi Ke Da Xue Xue Bao, 2016,36(2):151-156.

22. Singanayagam A, Manalan K, Connell D W, et al.Evaluation of serum inflammatory biomarkers as predictors of treatment outcome in pulmonary tuberculosis. Int J Tuberc Lung Dis, 2016,20(12):16531660.

23. Yang Z, Yu M, Li G, et al.Clinical Characteristics, Serological and Radiological Longitudinal Changes of Patients with COVID-19.Clin Lab, 2021,67(4).

24. World Health Organization, "Labratory Guidelines for enumerating CD4 T Lymphoctes in the context of HIV/AIDS," 2007, http://www.who.int/hiv/amds/LaboratoryGuideEnumeratingCD4TLymphocytes.pdf.

25. AIDS and Hepatitis C Professional Group, Society of Infectious Diseases;Chinese Medical Association Chinese Center for Disease Control and Prevention, Guidelines for AIDS diagnosis and treatment in China (2018 Edition). Electronic Journal of emerging infectious diseases, 2019,4 (02): 65-84

26. A. G. Alamdo, T. Fiseha, A. Tesfay, M. K. Deber, Z. M. Tirfe, and T. Tilahun, Anemia and its associated risk factors at the time of antiretroviral therapy initiation in public health facilities of Arba Minch Town, Southern Ethiopia, Health, vol. 07, no. 12, pp. 1657-1664, 2015.

27. Bruno G, Saracino A, Monno L, et al. The Revival of an "Old" Marker: CD4/CD8 Ratio. AIDS Rev, 2017,19(2):81-88 
28. Monsalvo $\mathrm{M}$, Vallejo A, Fontecha $\mathrm{M}$, et al. CD4/CD8 ratio improvement in HIV-1-infected patients receiving dual antiretroviral treatment. Int J STD AIDS, 2019,30(7):656-662.

29. Hu S, Rayman M P. Multiple Nutritional Factors and the Risk of Hashimoto's Thyroiditis. Thyroid, 2017,27(5):597-610.

30. Turker F, Oral A, Sahin T, et al. Does the FT3-to-FT4 ratio easily predict the progression of NAFLD and NASH cirrhosis?. J Int Med Res, 2021,49(11):675849527.

31. Beyene H B, Tadesse M, Disassa $H$, et al. Concurrent Plasmodium infection, anemia and their correlates among newly diagnosed people living with HIV/AIDS in Northern Ethiopia. Acta Trop, 2017,169:8-13.

32. Serrano-Villar S, Deeks S G. CD4/CD8 ratio: an emerging biomarker for HIV. Lancet HIV, 2015,2(3):e76-e77.

33. Mussini C, Lorenzini P, Cozzi-Lepri A, et al. CD4/CD8 ratio normalisation and non-AIDS-related events in individuals with HIV who achieve viral load suppression with antiretroviral therapy: an observational cohort study. Lancet HIV, 2015,2(3):e98-e106.

34. Ezeamama A E, Guwatudde D, Sikorskii A, et al. Impaired Hematologic Status in Relation to Clinical Outcomes among HIV-Infected Adults from Uganda: A Prospective Cohort Study. Nutrients, 2018,10(4).

35. Denz H, Orth B, Huber P, Gallati H, Wachter H, Fuchs D. Immune activation and anemia of chronic disorders. Blood. 1993 Mar 1;81(5):1404.

\section{Tables}

Tables 1 to 3 are only available as a download in the Supplemental Files section.

\section{Figures}


Figure 1

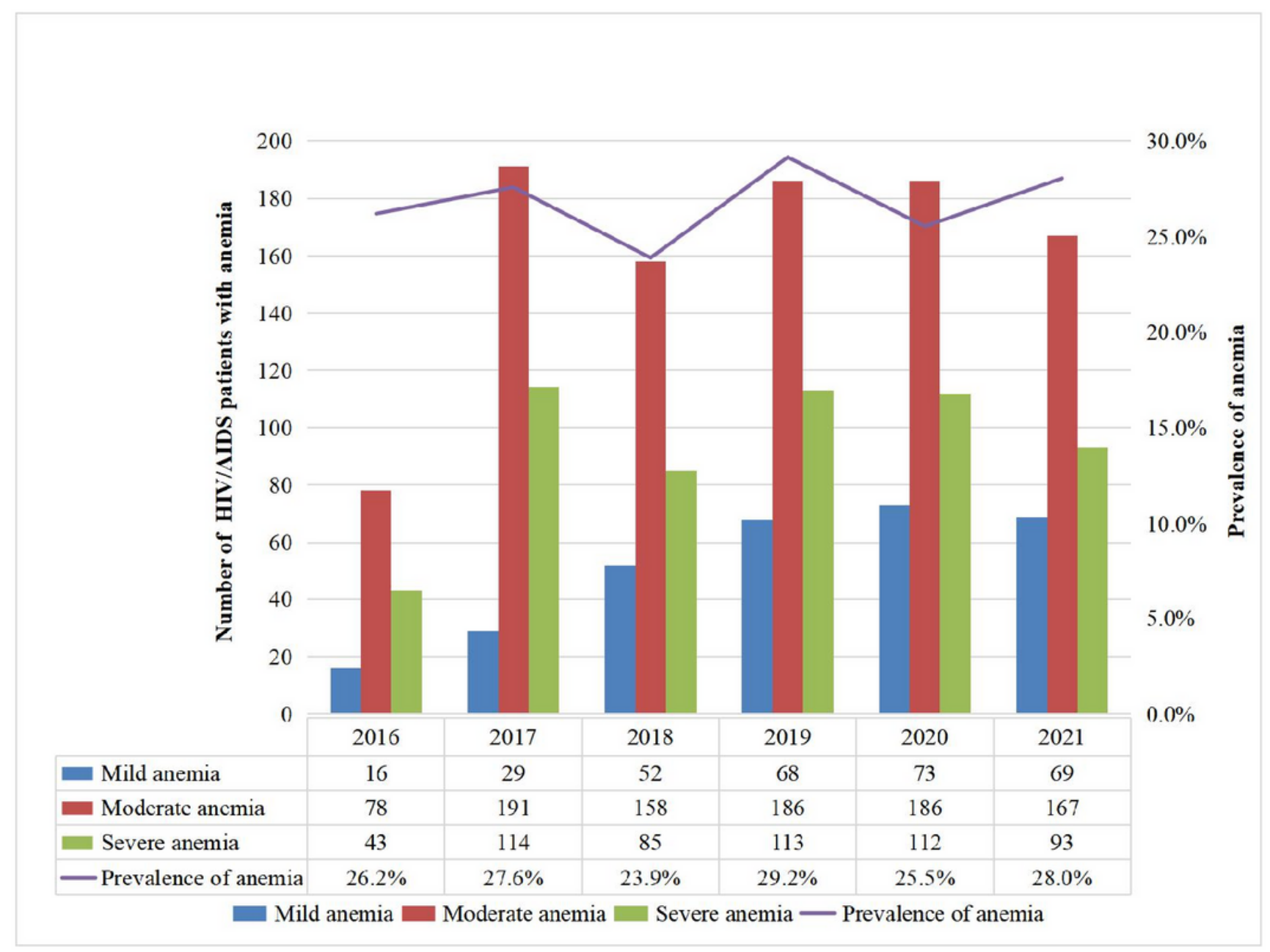

Figure 1

Number and prevalence of anemia in hospitalized HIV/AIDS from 2016 to 2021 
Figure 2

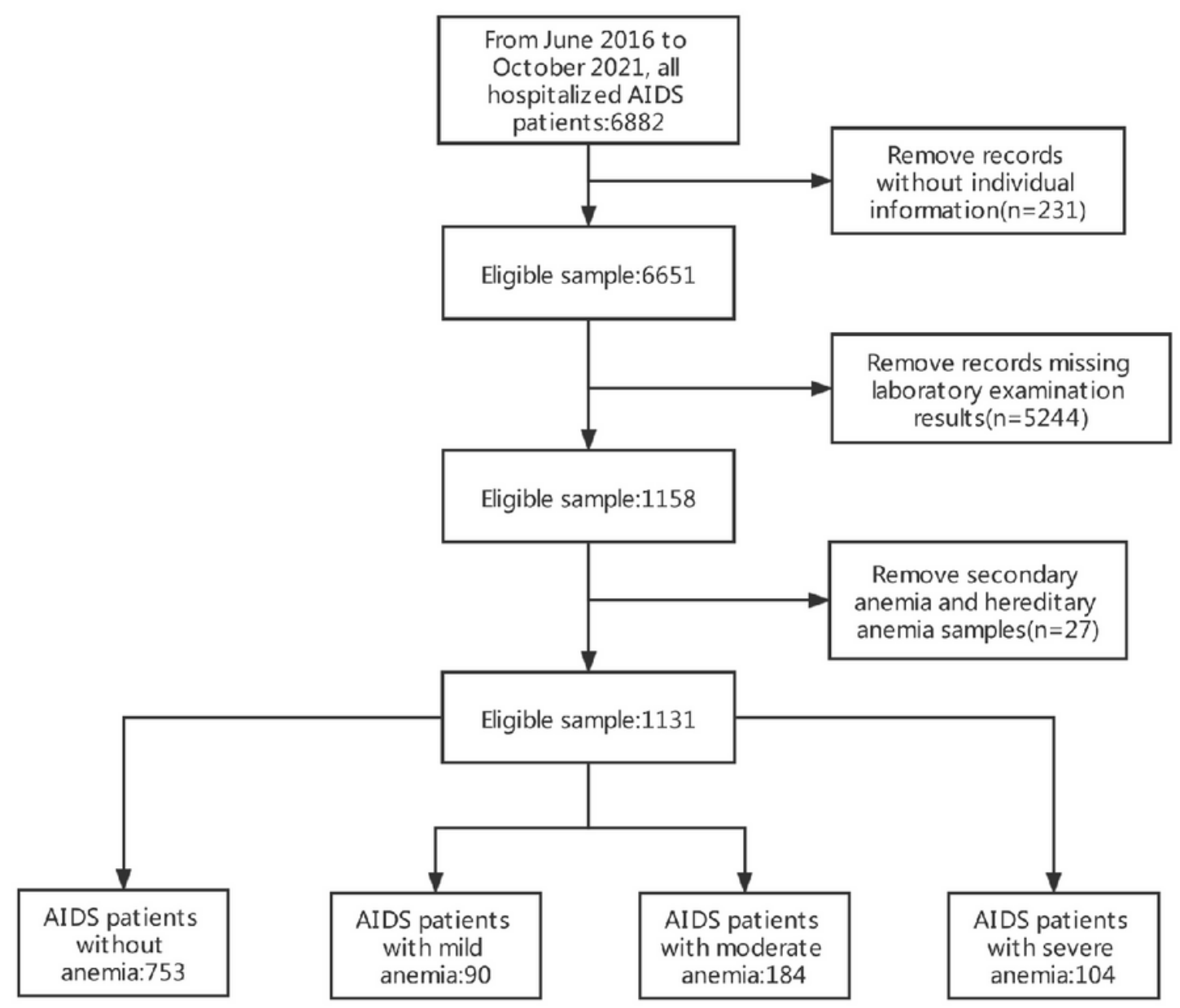

Figure 2

Inclusion and exclusion criteria of study participants

\section{Supplementary Files}

This is a list of supplementary files associated with this preprint. Click to download.

- Table.pdf 